\title{
FACTORES QUE INFLUENCIAN UN ESTILO DE VIDA SEDENTARIO EN LAS ESTUDIANTES DE CUARTO CICLO DE LOS COLEGIOS PÚBLICOS
}

\section{FACTORS THAT INFLUENCE IN SEDENTARY LIFESTYLE OF STUDENTS OF FOURTH CYCLE OF A PUBLIC HIGH SCHOOL}

\author{
Carlos Álvarez Bogantes ${ }^{1}$ \\ Escuela de Ciencias del Movimiento Humano y Calidad de Vida \\ Universidad Nacional. \\ ceab.03@gmail.com
}

\begin{abstract}
Resumen
Existe suficiente evidencia para afirmar que las mujeres, conforme avanzan en el sistema educativo, disminuyen la actividad física hasta niveles considerables de sedentarismo. Lo anterior condujo a determinar, como propósito de este artículo, los factores que influencian la inactividad física (sedentarismo) de un grupo de mujeres de cuarto ciclo de la educación pública costarricense. Para ello se diseña un estudio de corte cualitativo utilizando la técnica de grupos focales y las entrevistas a profundidad, la cual fue aplicada a 30 mujeres sedentarias de cuarto ciclo. Los resultados indican que las participantes desconocen el beneficio que les puede otorgar una vida activa, afectando posiblemente su conducta de movimiento Se concluye que la orientación deportiva de las clases de Educación Física, la carencia de las destrezas para participar en actividades en forma exitosa, las actividades sedentarias del círculo de amistades, las actitudes de los padres, la falta de opciones en el entorno y la carencia de instalaciones juegan un papel importante en los niveles de sedentarismo del grupo estudiado.
\end{abstract}

Palabras Claves: Actividad física, sedentarismo, educación secundaria, mujeres, barreras, creencias

\begin{abstract}
There is sufficient evidence to say that women as they move into the education system to reduce physical activity levels of sedentary lifestyle that put them at higher risk for non-communicable diseases. This led to determine the reasons for the inactivity of a group of fourth cycle. In order to address this problem, a qualitative design using focus groups and depth interviews was used, applied to30 women of high school participated. The results indicate that the participants are unaware of the benefits that can give them an active life, possibly affecting their movement behavior. A key element that have expressed is little impact of physical education classes when promoting lifestyles movement of the participants in this study, especially for ignoring the needs and barriers that students have. Become clear that the sport orientation of physical education classes and lack the skills to participate in activities successfully, sedentary activities of friends, the attitudes of parents; curriculum and lack of facilities also have significant impact in the studied group.
\end{abstract}

Key Words: Physical activity, sedentary lifestyle, high school, women, barriers, beliefs, 


\section{Introducción}

La inactividad física es uno de los factores líderes modificables para el riesgo de mortalidad y morbilidad entre los adultos. Los patrones de sedentarismo en la vida adulta usualmente son establecidos durante la adolescencia (Telana, Yang, Vilikan, Wanne y Raitakari, 2005), haciendo de este periodo de vida determinante para la promoción de la actividad física. Adicionalmente, está bien establecido en la literatura que las adolescentes son menos activas que los muchachos de su misma edad (Currie, Molcho y Boyce, 2008) y presentan niveles más pronunciados de sedentarismo durante la adolescencia (Kahn, Huang y Gillman, 2008; Sallis, 2000).

Según la Encuesta Global de Salud Escolar del Ministerio de Salud (2010), en Costa Rica solo un 27,4 \% de la población adolescente entre 13 a 15 años de edad es físicamente activa, al lograr cumplir con las recomendaciones de realizar 60 minutos o más de actividad física diaria. A su vez, menos de la mitad de esta población adolescente (43\%) no se moviliza en forma activa para desplazarse de la casa al colegio (en bicicleta o caminando) y un $44 \%$ pasa 3 horas o más sentados, fuera de las horas de colegio y de hacer tareas, por lo que se puede deducir que las actividades recreativas son de tipo sedentario (Ministerio de Salud, 2010). No obstante, los beneficios de la actividad física regular han sido claramente establecidos (WHO/ Fédération Internationale De Médecine Du Sport - Committee On Physical Activity For Health, 1995), por lo que la participación regular en actividad física está asociada con una calidad de vida mejor y más larga, reduciendo los riesgos de una variedad de enfermedades (Sallis y Owen, 1999).

La actividad física puede influenciar la salud de las adolescentes al impactar las causas de la enfermedad durante la niñez y la adolescencia. La evidencia sugiere una positiva relación entre la actividad física y los factores que afectan la salud de las adolescentes, reduciendo el riesgo de enfermedades crónicas (Sabo, Miller, Melnick, y Heywood, 2004) y una serie de enfermedades como cáncer, diabetes y enfermedad coronaria, que inician en la niñez (Freedman, Khan, Dietz, Srinivasan y Berenson, 2001). Es un hecho que si la actividad física inicia en la niñez y se mantiene a través de la vida, previene la osteoporosis, la cual afecta a las mujeres en forma predominante (Kannus, 1999).

A pesar del culto a la belleza que ha regido esta sociedad globalizada y a la importancia de la actividad física en relación con la prevención de las enfermedades cardio-degenerativas (WHO, 1999; United State Department of Health and Human Services [USDHHS], 1999), el sistema educativo no ha propiciado estilos de vida saludables entre los estudiantes, y especialmente entre las jóvenes de secundaria, las cuales no presentan los patrones de movimiento que se requiere para obtener beneficios para la salud (Fernández et al., 1998; Álvarez, 2012). Se ha reconocido que la inactividad física o sedentarismo es el factor de riesgo de enfermedad cardio-degenerativa más importante. Existe una cantidad significativa de literatura que muestra que la inactividad física es una de las variables más significativas en las causas de muerte, inhabilidad y en la reducción de la calidad de vida (USDHS, 1996). En el caso de las mujeres, es reconocido que factores biológicos, ambientales y sociales las predisponen a ser sedentarias y a enfrentar enfermedades 
cardio-degenerativas, osteoporosis, diabetes, obesidad y a desórdenes alimenticios, situaciones que tienen su inicio a edades tempranas (Shephard y Trudeau. 2000). Esta situación es especialmente preocupante en el caso de las mujeres adolescentes que presentan historial de inactividad durante su niñez, por el hecho de que ellas tendrán una posibilidad mayor de ser inactivas en su vida adulta, lo que hace que esta población sea una prioridad en el sistema educativo costarricense.

La participación en actividades físicas y deporte tiende a disminuir con la edad, particularmente durante la adolescencia; en el caso de las mujeres, la tendencia es a abandonar la participación deportiva a una edad más temprana que los muchachos. Esto hace que haya una gran necesidad de determinar las barreras potenciales y los motivadores que provocan esta situación. Recientemente, algunos estudios han explorado los factores que podrían influenciar la participación de las mujeres en actividades físicas y los elementos que propician la disminución de su participación (Slater y Tiggeman, 2011). Los resultados de la investigación mostraron que las mujeres son sometidas a burlas debido a su descoordinación y que esto podría generar los problemas con la imagen corporal que las adolescentes mostraron. En la misma línea, Dudley, Okely, Philip, Pearson y Peat (2011) encontraron que los programas escolares de movimiento humano que ponen poco énfasis en el área deportiva y se focalizan más en danza y ejercicios aeróbicos producen mayor participación de las adolescentes debido a que producen mayor disfrute.

A pesar de la continua inversión en la promoción de la actividad física y los cambios recientes en los programas de educación física, las jóvenes son inactivas, y es la carencia de destrezas uno de los detonantes para que ellas no se involucren en actividades físicas de por vida, lo que las predispone a enfrentar mayor riesgo de sufrir enfermedad coronaria (Álvarez, 2012; Ureña, Araya, Sánchez, Salas y Blanco, 2010; Sabo, Miller, Melnick y Heywood, 2004).

Desde una perspectiva de educación para la salud, el objetivo de este estudio fue el identificar las creencias, actitudes y percepciones de las estudiantes de cuarto ciclo en relación con su inactividad física. Fundamentalmente, este estudio, con un enfoque cualitativo, trató de focalizar una serie de percepciones, preferencias y creencias que son capaces de ampliar la comprensión teórica sobre el estilo de vida sedentario de las participantes. Esto posibilita identificar determinantes potencialmente modificables para la atención en intervenciones de salud a nivel de cuarto ciclo. Los resultados se enmarcan dentro de las temáticas de: conocimiento, creencias en salud, aptitudes hacia la Educación Física y las barreras que enfrentan para poder ser activas.

\section{Metodología}

\section{Participantes}

De cuatro colegios del cantón central de Heredia, Costa Rica, fueron seleccionados dos por criterio de conveniencia. Además, del cuarto ciclo de cada colegio se seleccionaron treinta estudiantes sedentarias, escogidas siguiendo los patrones de movimiento en un recordatorio de 
24 horas. Respondiendo a criterios de representatividad, se seleccionaron casos extremos que otorgaran la heterogeneidad buscada.

\section{Instrumentos}

Se aplicaron entrevistas y grupos focales. Todas las entrevistas y grupos focales con las alumnas fueron realizados en lugares privados seleccionados por las instituciones participantes, siguiendo un cuestionario de preguntas abiertas y con una duración estimada de quince a veinte minutos. Cada entrevista contempló al inicio preguntas sobre la opinión del grupo, esto con el fin de que se sintieran más relajadas o menos asediadas por preguntas directas. Antes del inicio de la entrevista, se le explicó a cada una de las participantes lo siguiente: el objetivo de la investigación, el hecho de que lo que expresaran sería grabado y de que todo lo que mencionaran se mantendría en absoluta confidencialidad. Además, se les explicaron los términos actividad física, ejercicio y deporte, con el fin de que comprendan a plenitud las preguntas.

Las observaciones que se hicieron correspondieron a situaciones clásicas de la clase de Educación Física, las cuales son: el calentamiento, la instrucción, el tiempo libre y la práctica de un colectivo. Todas las observaciones fueron realizadas sin previo aviso, para poder captar las conductas usuales de las estudiantes. En este punto fue indispensable incluir todos aquellos momentos en los cuales se pudiera ver las diferentes reacciones de movimiento de las alumnas y las respectivas reacciones para activarse o desactivarse físicamente.

\section{Procedimiento}

Para el estudio se seleccionó un diseño cualitativo interpretativo, ya que es la forma más cercana de acceder al pensar de las participantes y de obtener información que pueda realimentar la propuesta educativa que emana del Ministerio de Educación Pública, para que se puedan hacer los ajustes pertinentes en el currículo de cuarto ciclo, si fuera necesario. Con el propósito de poder obtener una visión más amplia de la realidad estudiada, se siguió la propuesta de triangulación de Denzin (2000), por lo que los métodos utilizados fueron las entrevistas personales grabadas (Taylor y Bogdan, 1992), los grupos focales y la observación realizada por al menos dos investigadores (Cutforth y Parker, 1996).

\section{Análisis estadístico}

El proceso de análisis de datos quedó configurado en torno a tres aspectos clave: reducción de datos, disposición y transformación de estos y, por último, la obtención de resultados y la verificación de conclusiones, según lo presentado por Rodríguez, Gil y García (1996).

La transcripción de las grabaciones, la confección de los memos y de las matrices se realizó inmediatamente después de que sucedieron los hechos. La confección de las matrices tuvo como 
URL: http://www.revistas.una.ac.cr/mhsalud

objetivo fundamental el encontrar patrones, comparaciones, tendencias y opuestos. Durante el proceso de investigación se realizó una revisión de la información recopilada por los investigadores, de lo cual surgieron nuevas preguntas que fueron integradas dentro de la investigación.

\section{Resultados}

El énfasis de este estudio fue explorar las creencias, actitudes y percepciones de las estudiantes de cuarto ciclo en relación con su inactividad física, para determinar factores personales y ambientales que puedan guiar el desarrollo de intervenciones que promuevan estilos de vida activos a través de la vida, con el propósito de que sirvan de insumo para cambios curriculares. Tres grandes tópicos están reportados en esta presentación de resultados: creencias en salud y actividad física, percepción de las barreras que enfrentan para hacer actividad física y las percepciones con respecto a la Educación Física que reciben en secundaria.

Sobre el primer aspecto, el exploró las creencias de las estudiantes sobre la salud y su relación con la actividad física. Las respuestas de las jóvenes muestran no solo un desconocimiento acerca de la importancia de la actividad física en relación con la salud, sino también la percepción de que, a sus edades, para ellas no es tan necesaria la actividad física, dejando claro que las personas mayores son los que se beneficiarían más de ser activos. Las siguientes expresiones ilustran cómo las estudiantes relacionan la actividad física con la salud: "claro que la actividad física tiene que ver con la salud, pero no es tan importante en este momento", "imagino que deberíamos pensar que la salud es un tesoro y que la actividad física ayuda, pero yo estoy saludable", "mi mamá siempre habla para que haga ejercicio, pero eso es para ellas". Otras expresiones como: "Siempre nos han martillado la importancia de la actividad física", "quién no sabe que es bueno para crecer saludables", presentan el pensar de ellas de que la actividad física es apropiada para la salud. Las participantes en este estudio han visto la necesidad de realizar actividad física o ejercicio a futuro. Además, queda evidenciado en este segmento colegial que el manejo teórico no garantiza un cambio de conducta hacia estilos de vida en movimiento.

En este grupo de estudiantes es notoria la confusión en el uso de los conceptos de actividad física, ejercicio y deporte, pero también el desconocimiento del tipo de actividad, tiempo e intensidad que se requiere para cumplir los estándares recomendados para obtener beneficios para la salud. Fue común escuchar: "es como hacer deporte", "no solo deporte"; y una expresión que fue compartida por la mayoría fue: "no solo es ir al gimnasio".

A pesar de la vaguedad de las respuestas en cuanto a la cantidad de actividad física requerida para la salud, en general las participantes expresaron que dependía de la persona y de la actividad física a realizar. Esta uniformidad o generalización fue reforzada al escuchar expresiones como: "pienso que hay que moverse" o "de seguro depende del organismo". En el momento que se les explicó las diferencias entre actividad física, ejercicio y deporte, se presentaron expresiones como: "ah pienso que no sería difícil ser activa" o "entonces soy algo

Revista MHSalud® (ISSN: 1659-097X) Vol. 12. No. 1. Febrero-Agosto, 2015 
activa". Otro grupo de mujeres se identificaron con las mujeres que hacen actividad física o ejercicio. Por ejemplo, gran parte de las jóvenes expresaron algún tipo de admiración por la mujer activa y la describen como alguien en buena forma: "pienso que ellas tienen más energía y pueden hacer cosas que yo no puedo".

Con gran sorpresa, algunas de las participantes expresaron no ser sedentarias, pero al presentarles los valores apropiados para la salud, se escucharon expresiones como: “ $\mathrm{AAh}$, eso parece demasiado para cualquiera!", "una hora es imposible que lo haga" o "no soy tan activa como pensaba". A partir de este punto, las muchachas hablaron con gran propiedad de la posibilidad de ser activas, "necesito solo un poco de movimiento para estar fit" o "fácilmente me pondría en forma”. Entre las que se consideran más activas del grupo, sin serlo, prevaleció la idea de moverse para conservar o mejorar el físico y fue común escuchar: "yo me muevo para lucir mejor" o "tenemos que cuidarnos para mantenernos".

Una mayoría de las entrevistadas manifiestan que la familia juega un rol determinante en el deseo de ellas para realizar actividad física. Para algunas fue importante ver a sus padres o a algún familiar haciendo actividad física. Por ejemplo: "vamos, apague el televisor, es lo que mi padre insiste conmigo" o "hoy toca caminar y espero que nos acompañe, menciona mi tía de vez en cuando".

Ante la apatía de realizar actividad física de las participantes, el grupo de amigas juega un rol importante para realizar algún tipo de actividad física a través de reuniones informales con el fin de socializar. Expresiones como "nos reunimos a bailar y terminamos hablando" o "al juntarnos oímos música, cantamos y nos movemos".

A diferencia de algunas entrevistadas que expresan que sus compañeros las ven como perezosas o vagas, algunas muchachas sienten apoyo en los compañeros de clase, especialmente apoyo verbal. Por ejemplo: "vengan a jugar" o "ellos nos ayudan en las clases de Educación Física a mejorar".

Cuando las instituciones promovieron actividades físicas pensando en ellas, las muchachas dijeron que: "la próxima semana tenemos que participar en un Zumba resistencia" o "mis amigos y yo nos preparamos para una carrera del cole"; sin embargo, pareciera que la motivación de ellas es fugaz y está determinada por la de percibirse competentes, como lo mencionó una de ellas, "tal vez haríamos más cosas, pero no sabemos cómo hacerlas".

La mayoría de las muchachas entrevistadas han desarrollado un sentido de indiferencia hacia la actividad física en sus vidas, siendo el entorno de secundaria un precursor de esa actitud. Ellas mencionan que en el ambiente colegial no se promueve su participación en actividades físicas, ya que lo que se ofrece no responde a las necesidades e intereses de ellas. Esto se ve reforzado en los siguientes comentarios: "nos gusta la música y el baile y es algo que no se ofrece en el cole" o "pensaría que en los recreos podrían dar Zumba o pirámides en el gym".

En lo que concierne ideas sobre educación física, la mayoría de las veces las participantes manifestaron que asisten a las clases de Educación Física para cumplir. Una de las alumnas, que 
solía jugar voleibol en años anteriores, manifestó: "no me disgusta hacer deporte, ya que estoy en juegos nacionales, pero la clase es aburrida”. Pareciera que las clases de Educación Física no generan niveles de motivación para participar en ellas. Algunas de las estudiantes manifestaron lo siguiente: "siempre haciendo la misma cosa de todos los años", o bien, que "desde sétimo ellas están sometidas a un régimen de repetición de actividades todos los años". Otra de las alumnas dijo: "mis profesores no nos presentan cosas nuevas". Esta idea se refuerza con otro comentario: "mis profesores de educación física son unos quitados, que solo baloncesto y voleibol nos dicen que vamos a aprender y nunca hemos aprendido nada".

El hecho de que el énfasis de la Educación Física lo siguen teniendo los deportes fue uno de los elementos que criticaron las entrevistadas. Una de las alumnas más comunicativa del grupo expresó: "desearía que se nos enseñaran cosas diferentes". Aunque esta alumna mostró una insatisfacción clara y directa de los contenidos, otra participante fue más drástica y dijo: "cuanto daría por recibir clases de Zumba". Evidentemente, estas alumnas manifiestan lo contrario de lo que han expresado alumnos en grados inferiores, los cuales catalogan a la lección de Educación Física como una de las clases que prefieren y disfrutan más.

Pareciera que las alumnas, conforme avanzan en el sistema educativo, pierden el atractivo por las actividades clásicas de edades inferiores, lo que propicia que desarrollen diferentes estrategias para evadir la clase de Educación Física, como nos dijo Laura: "la mejor forma de justificar mis ausencias a Educación Física es decir que tengo la regla". Aunque esta es una de las excusas de un grupo pequeño, otras recurren a citas médicas o a ausentarse durante época de exámenes.

Los siguientes comentarios reflejan la insatisfacción que tienen las estudiantes con la clase de Educación Física: "Baloncesto, voleibol y correr, eso es todo lo que hacemos en Educación Física" y "el profesor nos dice lo que debemos hacer... no lo que realmente queremos".

En el tema de barreras, una gran cantidad de creencias y actitudes fueron expresadas como barreras para hacer algún tipo de actividad física, algunas de las cuales ya se han mencionado; sin embargo, surgieron algunas barreras que vale la pena mencionar.

Una de las razones primordiales para no hacer actividad física fue el hecho de no desear hacer nada. "No estoy motivada" o "no tengo necesidad de hacer nada" fueron las palabras dichas por muchas de ellas. Lo anterior fue reforzado por palabras como "no hay tiempo", "tengo que cumplir en el cole".

Aunque no fue la constante en el grupo de alumnas, algunas consideran que los hombres pueden ser una barrera, ya que son poco tolerantes a las pocas destrezas de ellas y dan comentarios negativos cuando ellas cometen errores al practicar deportes en la clase de Educación Física. Expresiones negativas como: "son muy competitivos y nos aíslan, generando que algunas muchachas rehúyan involucrarse en actividades físicas organizadas en el contexto de Educación Física”.

Una de las barreras para no hacer actividad física, mencionada numerosamente, es la percepción de esfuerzo que requiere la actividad física, con tan poca retribución que obtienen, 
que se representa en forma apropiada con las expresiones: "qué aburrido", "quita mucho tiempo" o "será necesario hacerlo". Estas manifestaciones lo que muestran es un letargo para moverse, que también se refleja en las clases de Educación Física. Aunado a lo anterior, algunas frases expresadas por ellas detonan falta de compromiso o carencia de destrezas conductuales que les permitan hacer actividad física, lo cual se refleja por lo dicho por algunas de las alumnas: "lo he intentado por un par de veces y lo abandono", "rápidamente me aburro" o "los hombres mejenguean y nosotras hablamos" o "hemos hecho cosas bonitas en la clase de Educación Física, pero pronto regresamos a la rutina".

Cuando se les preguntó si realizaban actividad física en su tiempo libre, la mayoría manifestó el carecer de tiempo, debido a las exigencias académicas. Algunas dijeron que la inseguridad las limitaba, como dijo Rocío: "en la cancha del barrio llegan algunos mariguanos, lo que aleja a la gente del lugar".

Aunque algunas participantes mostraron deseos de hacer un poco más de actividad en el tiempo libre, la inseguridad es un factor que las aleja. Expresiones de los hombres en las calles como: "no me pueden ver en short porque me gritan cosas", las alejan de hacer actividad física. Sin embargo, la mayoría de las participantes mencionaron realizar actividades sedentarias. El escuchar música o ver televisión fueron mencionadas como las actividades más comunes en las estudiantes.

Por otro lado, la falta de un círculo social de amigos y amigas para hacer actividad física ha sido mencionada como una de las causas para mantenerse inactivas en el tiempo libre. Algunas de las participantes han mencionado que: "no tengo a nadie", "mis amigas andan en otras", "se requiere de compañía para que no sea aburrido" o "raramente mis padres me motivan a realizar actividad física".

Percibirse sin las destrezas necesarias para involucrarse en actividad física o mostrar falta de confianza en hacer algún tipo de actividad física o exponerse a ejecutar alguna actividad física, sin saber cómo hacerla, fue mencionada en forma reiterada. "No me gusta practicar deportes porque me da vergüenza no poder hacer lo que me piden" o "no sé ningún deporte y no tengo las destrezas para hacerlo" son algunas de las respuestas que externaron las muchachas, lo cual muestra un factor adicional que contribuye a que las muchachas de este estudio muestren un estilo sedentario de vida.

Algunas de las estudiantes han enfatizado el exceso de trabajo académico que tienen que enfrentar cotidianamente, por lo que algunas frases que usan para no hacer actividad física son: "cuando uno estudia todo el día, prefiere al finalizar el día hacer cosas de poco esfuerzo", "llego demasiado cansada a la casa y lo que hago es coger el control y ver televisión" o "tengo cosas más importantes que hacer, como estudiar". Evidentemente, la actividad física no se ve como importante o se percibe como algo que requiere mucho esfuerzo.

Cuando se habla de las barreras de infraestructura, las alumnas mencionan que usualmente las instalaciones de la institución educativa no están disponibles para el uso fuera de la clase de Educación Física y en el caso de las comunitarias, ellas desconocen la existencia de facilidades 
para poder ir. La inseguridad al ir a algunas instalaciones comunitarias ha sido mencionada como un elemento que las mantiene alejadas. Las participantes expresaron lo siguiente, con respecto al tema anterior: "nunca he visto que el gym esté disponible para ir a hacer algo fuera de clase", "no me gusta correr peligros en esos lugares", "no conozco que ofrezcan nada en esos lugares" o "mis amigas no irían conmigo".

El sudar y no contar con instalaciones para bañarse fue otra de las barreras mencionadas en mayor cantidad de casos. Por ejemplo: "no me gusta hacer Educación Física porque no hay baños adecuados" o "el andar el resto del día sudada me da asco".

A nivel personal, para muchas de ellas, el hacer actividad física solas les da vergüenza. Esto se refleja en los comentarios: "yo no lo haría sola, entrar en un gimnasio y que todo mundo me mire" o "es un bañazo salir a caminar".

Algunas de las que mencionaron que no conocían los lugares para hacer actividad física en el barrio, expresaron la imposibilidad de poder pagar un gimnasio. La siguiente idea refleja el sentir de las entrevistadas: "me gustaría ir al gimnasio, pero debe ser caro recibir clases" o "a veces bailo sola en mi casa, pero sería difícil encontrar un lugar por aquí”.

\section{Discusión}

Fundamentalmente, este estudio, con un enfoque cualitativo, ha identificado una serie de percepciones, preferencias y creencias que son capaces de ampliar la comprensión teórica sobre el estilo de vida sedentario de las participantes, posibilitando identificar determinantes potencialmente modificables para la atención en intervenciones de salud a nivel de cuarto ciclo. Los resultados se enmarcan dentro de las temáticas de: conocimiento, creencias en salud, aptitudes hacia la Educación Física y las barreras que enfrentan para poder ser activas.

Dentro de las teorías del cambio de conducta escolar y colegial hacia estilos de vida saludables, se valora altamente el conocimiento inicial que tengan los estudiantes sobre la actividad física y la salud. Este asidero inicial puede ser la diferencia entre intervenciones poco significativas y las exitosas (Ward, Saunders y Felton, 2007). Este estudio ha mostrado que las participantes no tienen un conocimiento claro acerca del concepto de salud y de su relación con la actividad física. Aunque ellas mencionaron la existencia de una relación entre ambas áreas y que de alguna manera la actividad física contribuye a la salud, es evidente el conocimiento superficial acerca de la importancia de la actividad física en el proceso de construcción de un estilo saludable en el grupo de este estudio, lo que se podría ver como una carencia en la adopción de conductas activas. Ellas percibieron que los beneficios en salud podrían darse más a largo plazo y que por lo tanto serían sus padres los más beneficiados.

La edad es el factor biológico dominante de la actividad física en las mujeres, lo cual se hace evidente con el declive de los niveles de actividad física desde la niñez hasta la adolescencia; 
sin embargo, esta disminución no debe ser entendida únicamente en términos de influencias biológicas, ya que los niveles del incremento del sedentarismo deben ser entendidos como algo multifactorial (Freedman, Kettel Khan, Dietz, Srinivasan y Berenson, 2001).

En consonancia con varias investigaciones, las muchachas en este estudio externaron no tener una historia de participación en actividad física durante la infancia y la adolescencia. Esto les ofrece, de forma significativa, menos probabilidades de ser físicamente activas como adultos, situación particularmente preocupante que podría agravar su situación a futuro (Shephard y Trudeau, 2000; Tammelin, Nayha, Hills y Jarvelin, 2003; Wallace, 2003).

Uno de los puntos claves en esta investigación es el hecho de que las participantes expresaron que ellas harían actividad física si se tomara en cuenta sus necesidades e intereses. Al respecto, Kilborn (1999) apoya la idea de que si las mujeres jóvenes son escuchadas a la hora del diseño del tipo de actividad física a realizar, habrá más posibilidades de que participen voluntariamente. Ennis (2003) agrega que el mayor reto que enfrenta el sistema educativo al desarrollar propuestas curriculares contextualizadas que atiendan los niveles altos de sedentarismo en las alumnas es el de involucrar directamente a las actoras directas del proceso, con el fin de propiciar propuestas que respondan a las inclinaciones particulares de ellas.

Aunque las barreras tienden a ser un tema prioritario en la mayoría intervenciones (Brett, Heimendinger, Boender, Morin y Marshall, 2002), es importante reconocer los factores que facilitan o que podría utilizarse para facilitar las conductas activas en la población femenina. Hay varios factores que emergieron en este estudio, que fueron percibidos como disparadores de la motivación para que las alumnas piensen en activarse físicamente. Por ejemplo, las adolescentes de este estudio, en su mayoría identifican a un familiar como el factor más influyente en su decisión de ser físicamente activas, sin que esto significara el inicio de una conducta activa de parte de ellas. Los hallazgos de este estudio en cuanto a la influencia percibida de los padres, a la hora de que las mujeres se involucren en actividad física, son apoyados por varios estudios. Estos estudios muestran que las adolescentes tienden a ser más activas cuando los padres son activos y las apoyan para realizar actividad física (McGuire, Hannan, Neumark-Sztainer, Cossrow y Story, 2002; USDHHS, 1996).

En algunos estudios, se reporta que las mujeres sufrieron algún tipo de acoso, burla o abuso verbal al involucrarse en actividad física durante las clases de Educación Física, lo que produjo indisposición de ellas. Sin embargo, las participantes en esta investigación vieron a sus compañeros como elementos de apoyo durante las clases, exceptuando el espacio de práctica libre de la actividad física al final de la clase, ya que en esa esfera una gran mayoría de las mujeres se sintieron invisibilizadas (Slater y Tiggermann, 2011; Dudley et al., 2010).

Algunas de las explicaciones que las participantes dieron en este estudio para no hacer actividad física incluyeron: presiones académicas en la escuela, la insatisfacción con las clases de Educación Física de la escuela, la molestia de sentirse sudadas y el problema de no tener instalaciones apropiadas. Resumiendo estos datos, Sallis y Owen (1999) mencionan que las adolescentes no solo están adquiriendo patrones adultos de hábitos sedentarios, sino también 
están adquiriendo formas adultas de pensar en relación con el ejercicio. Esto incluye las razones para no ser activas, como por ejemplo el hecho de que las actividades físicas a menudo no son importantes en sus vidas, ya que su núcleo de compañeras las alienta a buscar otras actividades asociadas con sus percepciones preferidas de género.

Evidentemente, las participantes en esta investigación se perciben sin las destrezas para involucrarse en actividad física, especialmente durante las clases de Educación Física. Gálvez (2007) señala como motivos principales para no involucrarse en la actividad física el carecer de aptitudes físicas y considerar que el ejercicio no es atractivo para ellas. De esta manera, las razones para hacer o no actividad física pueden ser diversas y tienen una gran validez desde la perspectiva subjetiva de cada persona. Indiscutiblemente, cualquier cambio que se quiera introducir en cuanto a conductas activas de vida en las adolescentes implicará comprender las razones para generar una actitud positiva hacia el mismo.

A pesar de que las clases de Educación Física deben responder a una propuesta curricular actualizada, las alumnas siguen expresando que las mismas tienen un gran componente de repetición a través de los años en la parte deportiva, sin ser un elemento que incida en el aprendizaje de destrezas. A la Educación Física en el ámbito educativo se le reconoce el gran potencial que tienen de promover estilos de vida activos de por vida (Stone, McKenzie, Welk y Booth, 1998; Wechsler, Devereaux, Davis y Collins, 2000).

Es evidente que el grupo estudiado considera que las clases de Educación Física no son un elemento motivante para activarlas físicamente, en especial por estar orientadas al deporte. Al respecto, Kilborn (1999) apoya el hecho de que si las necesidades y los intereses de las alumnas son tomados en cuenta, ellas estarán gustosas de participar de manera activa. Sin embargo, los educadores físicos luchan con el reto de ofrecer programas de educación física relevantes para las alumnas en concordancia con los nuevos programas del Ministerio de Educación Pública.

Fraser-Thomas y Beaudoin (2004) mencionan que con solo ofrecer cambios en las actividades de los nuevos programas no se logra el cambio hacia estilos de vida activos en las alumnas, sino que es necesaria toda una estrategia a nivel ambiental y en la capacitación de los docentes. Mientras el nuevo currículo ofrece mayores posibilidades para que las alumnas sean expuestas a actividades que responden a sus necesidades y gustos, los cambios no se asumen automáticamente, lo que requiere de mayor acompañamiento de las instancias en educación. De acuerdo con Friedman (2002), las mujeres colegiales comienzan a desarrollar sus actitudes hacia la Educación Física antes de que el tercer ciclo de educación finalice, por lo que sería poco probable que ellas se involucren posteriormente, aunque se les ofrezca la posibilidad.

Al igual que es mencionado en la teoría social cognitiva (Bandura, 1986), el estímulo verbal y la participación activa de los miembros de la familia contribuyeron a generar algún nivel de deseo o intención para involucrarse en actividad física, aunque no necesariamente haya movido a las participantes a ser activas; sin embargo, el apoyo de los padres debe ser uno de los elementos a considerar en cualquier intervención que pretenda impulsar estilos de vida activos en muchachas 
adolescentes. En este sentido, Beets, Cardinal y Alderman (2010) mencionan que los padres son los principales impulsores o inhibidores de la participación de sus hijos en la actividad física, apoyando la actividad física de sus hijos a través de refuerzo directo e indirecto. Estos resultados están apoyados por otros estudios que también encontraron que las adolescentes tienden a ser más activas cuando sus padres y hermanos están activos, y cuando los padres apoyan su participación en actividades físicas (McGuire, Hannan, Neumark-Sztainer, Cossrow y Story, 2002, USDHHS, 1996).

Otras barreras para realizar actividad física para las alumnas incluyeron el tiempo dedicado a tareas y deberes académicos, la carencia de oportunidades de actividad física, y los costos. Tales hallazgos son paralelos a otras investigaciones publicadas que encontraron poco apoyo de los compañeros y la familia, la falta de oportunidad en el entorno escolar y, un bajo autoconcepto en relación con sus capacidades para ser activas físicamente (Culp, 1998).

Adicionalmente a la falta de un círculo social de amigos y amigas para hacer actividad física, también fue mencionada la falta de apoyo familiar, siendo este elemento asociado a mayores niveles de actividad física. La participación de los padres es particularmente relevante cuando se trata de la participación en actividad física en las mujeres adolescentes, lo que hace de la influencia parental uno de los factores a tomar en cuenta en futuras intervenciones con mujeres colegialas (Davidson, Cutting y Birch, 2003).

Los compañeros de clase de las participantes en este estudio ocuparon un espacio importante, ya que ellas expresaron satisfacción con ellos y se sintieron a gusto teniendo a los hombres como compañeros, especialmente en los momentos de las prácticas deportivas; sin embargo, en el momento de realizar colectivos o juegos informales deportivos, usualmente ellas eran ignoradas por los varones, optando por sentarse a hablar. Diferente de lo expresado por las participantes en este estudio, autores como Slater y Tiggerman (2011) han reportado la presencia de experiencias de burla de los muchachos hacia las mujeres mientras practicaban deportes. Específicamente, las alumnas fueron más propensas a sufrir burlas debido a su apariencia o su falta de destrezas. Esta situación condujo a sugerir que los altos niveles de burla podrían influenciar la decisión de las mujeres para involucrarse en actividades deportivas.

En el marco de la línea de hallazgos de esta investigación y lo expresado por Dudley et al. (2010), surge la recomendación de que en el desarrollo de futuras intervenciones en cuarto ciclo se debe considerar actividades que enfaticen el componente recreativo, con la participación de las alumnas en la toma de decisiones de las actividades.

Las muchachas externaron que el énfasis tan alto en actividades deportivas eran razones importantes para no ser más activas físicamente. De forma adicional, ellas mencionaron el poco dominio que ellas tenían de las actividades deportivas y que las mismas no ofrecían ninguna relevancia para la vida. La carencia de competencias personales externadas por las participantes para involucrarse en actividades físicas brinda un aporte esencial a los procesos educativos en cuarto ciclo en esta área.

Independientemente de los niveles de actividad física que manifestaron tener las participantes en este grupo, el aburrimiento y falta de manejo hacia el tipo de actividad física ofrecida a través 
de los años de secundaria las alejó de cualquier involucramiento de la actividad. Al respecto, Pate, Ward, Saunders, Felton, Dishman y Dowda (2005) nos recuerdan que el desarrollo de estilos de vida activos está determinado por el sentimiento de confianza y competencia.

Aunque el nuevo currículo para Educación Física muestra una propuesta menos inclinada hacia los deportes, con una mayor cantidad de actividades físicas más innovadoras, pareciera que todavía esta visión de las nuevas tendencias en el ámbito del movimiento humano no está desarrollando estilos de vida en movimiento en la población de este estudio. Fraser-Thomas y Beaudoin (2004) llaman la atención de que los cambios curriculares en Educación Física toman tiempo para que se implanten y requieren ser iniciados desde primer ciclo, ya que la construcción de las actitudes hacia el movimiento humano y la Educación Física inician desde los primeros años.

Si las estudiantes no encuentran valor en los programas de Educación Física desde edades tempranas, es poco probable que ellas tengan las destrezas motoras y conductuales para ser activas (Gibbons y Humbert, 2008). Al igual que las muchachas de este estudio, diversos autores han enfatizado la importancia de ofrecer programas basados en actividades de por vida, que ofrezcan disfrute y llenen las necesidades de las estudiantes con el objeto de responder a los niveles altos de sedentarismo en estas edades (Flintoff y Scraton, 2001; Gibbons, Wharf Higgins, Gaul y Van Gyn, 1999; Humbert, 1995).

\section{Conclusión y recomendaciones}

Este estudio ha puesto de relieve algunas evidencias útiles para entender los niveles de sedentarismo entre la población femenina de cuarto ciclo de colegio. Se desprende de este estudio que estas mujeres jóvenes se enfrentan a retos particulares en relación a desarrollar una vida activa que las conduzca a un estilo de vida saludable de por vida.

Es importante resumir que las participantes mostraron un desconocimiento evidente sobre los beneficios de una activa para su salud, la influencia del grupo de amigos y amigas no fue mencionado como detonador para realizar actividad física y, diferente a la anterior, la influencia parental representa una de las voces que es escuchada por las participantes a la hora de generar niveles de intención para logra activarse físicamente. Un elemento clave que han manifestado las alumnas es el poco impacto de las clases de Educación Física a la hora de promocionar estilos de vida en movimiento de las participantes en este estudio, sobre todo por ignorar las necesidades y barreras que las estudiantes de cuarto ciclo enfrentan.

Se concluye que las participantes en este estudio consideran que la orientación deportiva de las clases de Educación Física, la carencia de las destrezas para participar en actividades en forma exitosa, las actividades sedentarias del círculo de amigas, las actitudes de los padres y la del plan de estudios, y las instalaciones tienen un papel importante en los niveles de sedentarismo del grupo estudiado. 
Los resultados en este trabajo representan una base útil para las autoridades educativas del país, con el objeto de que puedan a integrar nuevos elementos dentro del currículo escolar que contribuyan a una mejor respuesta de las estudiantes en las clases de Educación Física y en la construcción de un estilo de vida activo de por vida.

\section{Referencias bibliográficas}

Álvarez, C. (2012). Impacto del modelo del "juego a la salud" en los estilos de vida saludables de estudiantes de primaria. Revista MHSalud, 9(2)1-10. Recuperado en http://www.revistas. una.ac.cr/index.php/mhsalud/article/view/5201

Bandura, A. (1986). Social Foundation of thought and action: A social cognitive-view. New York: Prentice Hall.

Beets, M.W., Cardinal, B. J., y Alderman, B. L. (2010) Parental social support and the physical activity-related behaviors of youth: A review. Health Education y Behavior, 37(5), 621-644. http://dx.doi.org/10.1177/1090198110363884

Brett, J.A, Heimendinger J, Boender C, Morin C, y Marshall J.A. (2001).Using ethnography to improve intervention design. American Journal of Health Promotion, 16(6), 331-340. http://dx.doi.org/10.4278/0890-1171-16.6.331

Culp, R. (1998). Adolescent girls and outdoor recreation: A case study examining constraints and effective programming. Journal of Leisure Research, 30(3), 356-379.

Currie, C., Molcho, M., y Boyce, W. (2008). Researching health inequalities in adolescents: the development of the Health Behaviour in School-aged Children(HBSC). Family Affluence Scale. Social and Science Medicine, 66,1429-36. http://dx.doi.org/10.1016/j.socscimed.2007.11.024

Cutforth, N. y Parker, M. (1996, September). The Value of Journal Writing. JOPERD, 67, 19-23.

Davison, K., Cutting, T., y Birch, L. (2003) Parents Activity-Related Parenting Practices Predict Girls, Physical Activity. Medicine \& Science in Sports \& Exercise, 35, 1589-1594.

Denzin, N., y Lincoln, Y. (2000). Handbook of qualitative research. 2nd ed. Thousand Oaks: Sage Publications.

Dudley, D., Okely, A., Pearson, P. y Peat, J. (2010). Engaging adolescent girls from linguistically diverse and low income backgrounds in school sport: A pilot randomized controlled trial. Obesity, 13, 217-224. http://dx.doi.org/10.1016/j.jsams.2009.04.008

Ennis, C. (2003). Using curriculum to enhance student learning. In S. J. Silverman y C. D. Ennis (Eds.), Student learning in physical education: Applying research to enhance instruction (2nd ed., pp. 109-127). Champaign, IL: Human Kinetics Publishers. 
Ernst, M., Pangrazi, R. y Corbin, C. (1998-March). Physical education: Making a transition toward activity. JOPERD, 69, 29-32.

Flintoff, A., y Scraton, S. (2001). Stepping into active leisure? Young women's perceptions of active lifestyles and their experiences of school physical education. Sport, Education and Society, 6(1), 5-21. http://dx.doi.org/10.1080/713696043

Fraser-Thomas y Beaudoin (2004). Girls appreciation of new physical education curriculum classes. AVANTE, 10, 45-56.

Fernández, A., Ulate, G., Romero, M., y Murillo, S. (1996). Factores de riesgo cardiovascular en estudiantes de 17-19 años de la Universidad de Costa Rica. Documento Técnico, Facultad de Medicina, Universidad de Costa Rica.

Freedman, D., Kettel Khan, L., Dietz, W., Srinivasan, S. y Berenson, G. (2001) Relationship of Childhood Obesity to Coronary Heart Disease Risk Factors in Adulthood: the Bogalusa Heart Study. Pediatrics, 108, 712-718. http://dx.doi.org/10.1542/peds.108.3.712

Friedman, S. S. (2002). Body thieves: Helping girls reclaim their natural bodies and become more physically active. Vancouver, BC: Salal Books.

Gálvez, A. (2007). Actividad física habitual de los adolescentes de la región de Murcia. Análisis de los motivos de práctica y abandono de la actividad físico-deportiva. Recuperado de http://www.efdeportes.com/efd107

Gibbons, S. and Humbert, L. (2008). What are middle-school girls looking for in Physical Education? Canadian Journal of Education, 31, 167-186.

Gibbons, S. L., Wharf Higgins, J., Gaul, C. A., y Van Gyn, G. H. (1999). Listening to female students in high school physical education. AVANTE, 5(2), 1-20.

Humbert, M. L. (1995). On the sidelines: The experiences of young women in physical education classes. AVANTE, 1(2), 58-77.

Kahn J.A, Huang B, y Gillman M.W. (2008). Patterns and determinants of physical activity in US adolescents. Journal of Adolescent Health, 42, 369-77. http://dx.doi.org/10.1016/j. jadohealth.2007.11.143

Kannus, P. (1999) Preventing Osteoporosis, Falls and Fractures among Elderly People. British Medical Journal, 318, 205-206. Xi http://dx.doi.org/10.1136/bmj.318.7178.205

Kilborn, M. (1999). Investigating female student enrollment in Physical Education 11. Unpublished master's thesis. University of Victoria Victoria, British Columbia. 
McGuire, M.T., Hannan, P.J., Neumark-Sztainer, D., Cossrow, N., y Story, M. (2002). Parental correlates of physical activity in a racially/ethnically diverse adolescent sample. Adolescent Health, 30, 253-261. http://dx.doi.org/10.1016/S1054-139X(01)00392-5

Ministerio de Salud (2010). Encuesta Global de Salud Escolar 2009. Global School-based Student Health Survey (GSHS), San José, Costa Rica.

Pate, R., Ward, D., Saunders, R., Felton, G., Dishman, R., y Dowda, M. (2005). Promotion of physical activity among high-school girls: A randomized controlled trial. American Journal of Public Health, 95, 1582-1587. http://dx.doi.org/10.2105/AJPH.2004.045807

Pate R.R, Freedson P.S, y Sallis J.F. (2002). Compliance with physical activity guidelines: prevalence in a population of children and youth. Ann Epidemiol, 12, 303-308. http://dx.doi.org/10.1016/S1047-2797(01)00263-0

Rodríguez, G., Gil, J. y García, E. (1996). Métodos de investigación cualitativa. Málaga, Ediciones Aljibe. Recuperado de http://148.202.18.157/sitios/catedrasnacionales/ material/2010b/ortiz/infmic.pdf

Sabo, D., Miller, K., Melnick, M. y Heywood, L. (2004). Her Life Depends On It: sport, physical activity and the health and well-being of American girls. East Meadow, US: Women's Sports Foundation. Deprés, J.-P.

Sallis, J. y Owen, N. (1999). Physical Activity and Behavioral Medicine. Thousand Oaks, US: Sage.

Slater, A. y Tiggemann, M. (2011). Gender differences in adolescent sport participation, teasing, self-objectification and body image concerns. Journal of Adolescence, 34, 455-63. http:// dx.doi.org/10.1016/j.adolescence.2010.06.007

Sallis J.F. (2000). Age-related decline in physical activity: a synthesis of human and animal studies. Med Sci Sports Exerc, 32, 1598-600. http://dx.doi.org/10.1097/00005768200009000-00012

Shephard, R. J., y Trudeau, F. (2000). The legacy of physical education: Influences on adult lifestyle. Pediatric Exercise Science, 12(1), 34-50.

Stone, E. J., McKenzie, T. L., Welk, G. J., y Booth, M. (1998). Effects of physical activity interventions in youth: Review and synthesis. American Journal of Preventive Medicine, 15(4), 298-315. http://dx.doi.org/10.1016/S0749-3797(98)00082-8

Tammelin, T., Nayha, S., Hills, A., y Jarvelin, M. (2003). Adolescent participation in sports and adult physical activity. American Journal of Preventive Medicine, 24(1), 22-28. http:// dx.doi.org/10.1016/S0749-3797(02)00575-5 
URL: http://www.revistas.una.ac.cr/mhsalud

Taylor, S. y Bogdan, R. . (1992). Introducción a los métodos cualitativos en investigación. La búsqueda de los significados. España: Paidós

Telana, R., Yang, X., Vilikan, L., Wanne, O. y Raitakari, O. (2005). Physical activity forms childhood a 21 year tracking study. American journal of preventive medicine, 28, 267-273. http://dx.doi.org/10.1016/j.amepre.2004.12.003

United States Department of Health and Human Services (USDHHS). (1996). Physical Activity and Health: a report of the Surgeon General. Atlanta, US: Centers for Disease Control.

United State Department of Health and Human Services. (USDHHS). (1999). Mental Health: $a$ report of the Surgeon General. Atlanta, US: Centers for Disease Control.

Wallace, L. S. (2003). Correlates of lifetime physical activity in young women. American Journal of Health Education, 34(1), 41-46. http://dx.doi.org/10.1080/19325037.2003.10603524

Ward, D.S., Saunders, R., Felton, G.M. (2007). Implementation of a school environment intervention to increase physical activity in high school girls. Health Education Research, 21, 896-910. http://dx.doi.org/10.1093/her/cyl134

Wechsler, H., Devereaux, R. S., Davis, M., y Collins, J. (2000). Using the school environment to promote physical activity and healthy eating. Preventive Medicine, 31(2), S121-S137. http:// dx.doi.org/10.1006/pmed.2000.0649

World Health Organization / Fédération Internationale De Médecine Du Sport - Committee On Physical Activity For Health (1995) Exercise for Health. Bulletin of the World Health Organization, 73(2), 135-136

World Health Organization. (1999). The World Health Report. Switzerland:WHO.

Recepción: 04 de marzo del 2015.

Corrección: 14 de julio del 2015.

Aceptación: 21 de julio del 2015.

Publicación: 21 de agosto del 2015.

1 El autor es Doctor en Educación por la Universidad Nacional de Costa Rica; Profesor de la Escuela de Ciencias del Movimiento Humano y Calidad de Vida en la Carrera de Promoción de la Salud Física y Especialista en Fisiología y Ejercicio. 